\title{
A Study of the Zarisky Topology through Functors
}

\author{
Dr. Pravanjan Kumar Rana
}

Department of Mathematics, Berhampore Girl's College, West Bengal ,India

Keywords: Affine algebraic set, affine variety, category, contravariant functor and natural transformation.

Abstract: The aim of this paper is to study the Zarisky topology through a functor. In this paper we construct

i) Zarisky topology in $\mathrm{K}^{\mathrm{n}}$ and a functor associated with this topology ;

ii) $\operatorname{Spec}(R)$ is a Zarisky topology in a ring $R$, where $\operatorname{Spec}(R)$ denotes the set of all prime ideals of $\mathrm{R}$ and construct a functor 'Spec' associated with Zarisky topology; and finally

iii) we study the functor 'Spec' associated with Zarisky topology

\section{Introduction}

The concept of affine algebraic sets plays an important role to construct Zarisky topology. In this section we give some basic definitions.

Definition 1.1 Let $\mathrm{K}\left[\mathrm{x}_{1}, \mathrm{x}_{2}, \mathrm{x}_{3}, \ldots, \mathrm{x}_{\mathrm{n}}\right]$ denotes the polynomial ring over an arbitrary field $\mathrm{K}$ in $\mathrm{n}$-variables and $\mathrm{f}_{1}, \mathrm{f}_{2}, \mathrm{f}_{3}, \ldots \ldots, \mathrm{f}_{\mathrm{m}} \in \mathrm{K}\left[\mathrm{x}_{1}, \mathrm{x}_{2}, \mathrm{x}_{3}, \ldots, \mathrm{x}_{\mathrm{n}}\right]$. The function $\mathrm{V}: \mathrm{S} \rightarrow \mathrm{K}^{\mathrm{n}}$ defined by $\mathrm{V}(\mathrm{S})=\left\{(\mathrm{x}) \in \mathrm{K}^{\mathrm{n}}: \mathrm{f}(\mathrm{x})=0, \quad \forall \mathrm{f} \in \mathrm{S}\right\}$, where $\mathrm{S}=\left\{\mathrm{f}_{1}, \mathrm{f}_{2}, \mathrm{f}_{3}, \ldots \ldots, \mathrm{f}_{\mathrm{m}}\right\} \subset \mathrm{K}\left[\mathrm{x}_{1}, \mathrm{x}_{2}, \mathrm{x}_{3}, \ldots, \mathrm{x}_{\mathrm{n}}\right]$ and $(\mathrm{x})=\left(\mathrm{x}_{1}, \mathrm{x}_{2}, \mathrm{x}_{3}, \ldots, \mathrm{x}_{\mathrm{n}}\right) \in \mathrm{K}^{\mathrm{n}}$ is called the algebraic set i.e., the algebraic set $\mathrm{V}(\mathrm{S})$ is the set of solutions in $\mathrm{K}^{\mathrm{n}}$ of the system of equations:

$$
\mathrm{f}_{1}\left(\mathrm{x}_{1}, \mathrm{x}_{2}, \mathrm{x}_{3}, \ldots, \mathrm{x}_{\mathrm{n}}\right)=0, \mathrm{f}_{2}\left(\mathrm{x}_{1}, \mathrm{x}_{2}, \mathrm{x}_{3}, \ldots, \mathrm{x}_{\mathrm{n}}\right)=0, \mathrm{f}_{3}\left(\mathrm{x}_{1}, \mathrm{x}_{2}, \mathrm{x}_{3}, \ldots, \mathrm{x}_{\mathrm{n}}\right)=0, \ldots, \mathrm{f}_{\mathrm{m}}\left(\mathrm{x}_{1}, \mathrm{x}_{2}, \mathrm{x}_{3}, \ldots, \mathrm{x}_{\mathrm{n}}\right)=0 .
$$

Definition 1.2 A subset $A$ in $K^{n}$ is called an affine algebraic set if $A=V(S)$ for some $S \subset K[x]$. Thus any algebraic set $A$ is defined by a finite set of polynomials in $\mathrm{K}$ [x].

Definition 1.3 An algebraic set $A$ in $K^{n}$ is called irreducible or an affine variety iff $A \neq B \cup C$, where $\mathrm{B}$ and $\mathrm{C}$ are algebraic sets in $\mathrm{K}^{\mathrm{n}}$ and $\mathrm{A} \neq \mathrm{B}, \mathrm{A} \neq \mathrm{C}$.

Definition 1.4 Let $\mathrm{k}$ be a subfield of $\mathrm{K}$. If $\mathrm{A}$ is an affine algebraic set in $\mathrm{K}^{\mathrm{n}}$ admits a set of generators in $\mathrm{k}\left[\mathrm{x}_{1}, \mathrm{x}_{2}, \mathrm{x}_{3}, \ldots, \mathrm{x}_{\mathrm{n}}\right] \subset \mathrm{K}\left[\mathrm{x}_{1}, \mathrm{x}_{2}, \mathrm{x}_{3}, \ldots, \mathrm{x}_{\mathrm{n}}\right]$, then $\mathrm{A}$ is called an affine $(\mathrm{K}, \mathrm{k})$ algebraic set and $\mathrm{k}$ is called the field of definition of $\mathrm{A}$. Thus an affine $(\mathrm{K}, \mathrm{k})$ algebraic set $\mathrm{A}$ is a subset in $\mathrm{K}^{\mathrm{n}}$ consisting of all common zeros of a subset of polynomials in $k\left[\mathrm{x}_{1}, \mathrm{x}_{2}, \mathrm{X}_{3}, \ldots, \mathrm{x}_{\mathrm{n}}\right]$.

If $\mathrm{k}=\mathrm{K}$, we call $\mathrm{A}$ is an absolute affine algebraic set in $\mathrm{K}^{\mathrm{n}}$.

Definition 1.5 A category $C$ consists of

a) a class of objects $X, Y, \ldots$,denoted by $\mathrm{Ob}(\boldsymbol{C})$;

b) for each ordered pair of objects $\mathrm{X}, \mathrm{Y}$ a set of morphisms with domain $\mathrm{X}$ and range $\mathrm{Y}$ denoted by $C(\mathrm{X}, \mathrm{Y})$

c) for each ordered triple of objects $X, Y$ and $Z$ and a pair of morphisms; $f: X \rightarrow Y$ and $g: Y \rightarrow Z$, their composite is denoted by $\mathrm{gf}: \mathrm{X} \rightarrow \mathrm{Z}$, satisfying the two axioms:
i) associativity
ii) identity

Definition 1.6 Let $\boldsymbol{C}$ and $\boldsymbol{D}$ be two categories. A contravariant functor T from $\boldsymbol{C}$ to $\boldsymbol{D}$ consists of

a) an object function which assigns to every object $\mathrm{X}$ of $\boldsymbol{C}$ and object T(X) of $\boldsymbol{D}$; and

b) a morphism function which assigns to every morphism $\mathrm{f}: \mathrm{X} \rightarrow \mathrm{Y}$ in $\boldsymbol{C}$, a morphism

$\mathrm{T}(\mathrm{f}): \mathrm{T}(\mathrm{Y}) \rightarrow \mathrm{T}(\mathrm{X})$ in $\boldsymbol{D}$ such that

i) $\mathrm{T}(\mathrm{IX})=\operatorname{IT}(X)$,

ii) $\mathrm{T}(\mathrm{g}$ f) $=\mathrm{T}(\mathrm{f}) \mathrm{T}(\mathrm{g})$, for $\mathrm{g}: \mathrm{Y} \rightarrow \mathrm{Z}$ in $\boldsymbol{C}$

Definition 1.7 Let $\mathbf{C}$ and $\mathbf{D}$ be categories. Suppose $T_{1}$ and $T_{2}$ are both contravariant functors from C to D. A natural transformation $\Phi$ from $T_{1}$ to $T_{2}$ is a function from the objects of $\mathbf{C}$ to the morphisms of $\mathbf{D}$ such that for every morphism $\mathrm{f}: \mathrm{X} \rightarrow \mathrm{Y}$ in $\mathbf{C}$ the following condition holds:

$\Phi(\mathrm{X}) \mathrm{T}_{1}(\mathrm{f})=\mathrm{T}_{2}(\mathrm{f}) \Phi(\mathrm{Y})$ 


\section{Elementary properties}

In this section we give some basic results which are essential in the sequel.

Lemma 2.1 A subset $U$ of $K^{n}$ is an open set iff $K^{n}$ - $U$ is an affine $k$ - algebraic set. Thus an affine $\mathrm{k}$-algebraic sets in $\mathrm{K}^{\mathrm{n}}$ are the closed sets in $\mathrm{K}^{\mathrm{n}}$.

Proof: Using [4] and [5], it follows.

Lemma 2.2 Any subset $A$ of $\mathrm{K}^{\mathrm{n}}$ is an affine k-variety, then a subset $\mathrm{A}$ is closed in $\mathrm{K}^{\mathrm{n}}$ iff it is an affine k-algebraic set.

Proof: Using Definition 1.2, Definition 1.4 and Lemma 2.1, it follows.

Lemma 2.3 Any finite subset of $\mathrm{K}^{\mathrm{n}}$ is an algebraic set.

Proof: Using [4, art.5.5], it follows.

Lemma 2.4 An algebraic set $A$ in $K^{n}$ is affine variety iff $I(A)$ is a prime ideal.

Proof: Using [4, art.5.5], it follows.

Lemma 2.5 Let $\mathbf{C}$ be any category and $\mathbf{T}$ be a contravariant functor from $\mathbf{C}$ to $\mathbf{S}$ (category of sets and functions). Then for any object $\mathbf{C}$ in $\mathbf{C}$, there is an equivalence

$\Omega:\left(h^{\mathbf{C}}, \mathbf{T}\right) \rightarrow \mathbf{T}(\mathrm{C})$, where $\left(\mathbf{h}^{\mathbf{C}}, \mathbf{T}\right)$ is the class of natural transformations from the set valued functor $\mathbf{h}^{\mathbf{C}}$ to the set valued functor $\mathbf{T}$ such that $\Omega$ is natural in $\mathbf{C}$ and $\mathbf{T}$.

Proof: From [4, appendix B], it follows.

Lemma 2.6 For each subset $P$ of a ring $R$, let $X=V(P)$ denotes the set of all prime ideals of $R$ which contains $P$, then

i) $\quad \mathrm{V}(0)=\mathrm{X}$ and $\mathrm{V}(1)=\varnothing$,

ii) If $\left(\mathrm{P}_{\mathrm{i}}\right)_{\mathrm{i} \in \mathrm{I}}$ is any family of subsets of prime ideals of $\mathrm{R}$, then

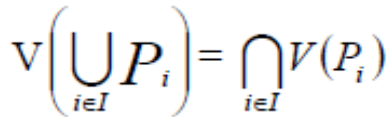

iii) $\quad \mathrm{V}\left(\mathrm{P}_{1}\right) \cup \mathrm{V}\left(\mathrm{P}_{2}\right)=\mathrm{V}\left(\mathrm{P}_{1} \cap \mathrm{P}_{2}\right)=\mathrm{V}\left(\mathrm{P}_{1} \mathrm{P}_{2}\right)$.

Proof: Using [4, art.5.5], it follows.

This shows that the set $\mathrm{V}(\mathrm{P})$ satisfies all the axioms for closed sets in a topological space.

Lemma 2.7 (Yoneda's Lemma) Let $\mathbf{C}$ be any category and $\mathbf{S}$ be the category of sets and functions.

Let $\mathrm{T}: \mathbf{C} \rightarrow \mathbf{S}$ be a contravariant functor. Then for any $\mathrm{C}$ in $\mathbf{C}$, there is an equivalence

$\Psi:\left(h^{\mathrm{C}}, \mathrm{T}\right) \rightarrow \mathrm{T}(\mathrm{C})$, where $\left(\mathrm{h}^{\mathrm{C}}, \mathrm{T}\right)$ is the class of natural transformations from the set valued functor $\mathrm{h}^{\mathrm{C}}$ to the set valued functor $\mathrm{T}$ such that $\Psi$ is natural in $\mathrm{C}$ and $\mathrm{T}$.

\section{Functor associated with Zarisky topology}

In this section we construct and investigate the Zarisky topology. To do this we prove the following:

Theorem 3.1 All affine k-algebraic sets in Knare the closed sets in $\mathrm{K}^{\mathrm{n}}$. Then this sets form a topology in $\mathrm{K}^{\mathrm{n}}$. This topology is called the Zarisky topology in $\mathrm{K}^{\mathrm{n}}$.

Proof: Since empty set and whole set are closed sets; the intersection of any family of closed sets is a closed set; and the union of two closed set is a closed set. Using Lemma 2.2, it follows.

Let $R$ be a ring. Define prime spectrum $\operatorname{Spec}(R)$ of $R$ by $X=\operatorname{Spec}(R)=\{P: P$ is prime ideal of $R\}$. Theorem 3.2 Let $\operatorname{Spec}(\mathrm{R})$ denotes the set of all prime ideals of a ring R. All subsets in the set of all prime ideals of a ring R, form a topology $\operatorname{Spec}(\mathrm{R})$, is called Zarisky topology.

Proof: Using Lemma 2.2, Lemma 2.5, Lemma 2.7 \& Theorem 3.1, it follows.

Theorem 3.3 Let $\mathbf{R}$ be the category of rings and ring homomorphisms and $\mathbf{T}$ denote the category of sets and functions. Then Spec: $\mathbf{R} \rightarrow \mathbf{T}$ is a contravariant functor

Proof: Let $\mathrm{R}$ and $\mathrm{T}$ are rings in $\mathbf{R}$ and $\mathrm{f}: \mathrm{R} \rightarrow \mathrm{T}$ is a ring homomorphism in $\mathbf{R}$. Let $\mathrm{X}=\mathbf{S p e c}(\mathrm{R})$ and $Y=\operatorname{Spec}(T)$. Define $f^{*}: Y \rightarrow X$ by $f^{*}(Q)=f^{-1}(Q), \forall Q \in Y$, then $f^{-1}(Q)$ is a prime ideal of $R$ and hence $\mathrm{f}^{-1}(\mathrm{Q}) \in \mathrm{X}$.

Let $f: R \rightarrow T$ and $g: T \rightarrow M$ be a ring homomorphisms in $R$. Then $g f: R \rightarrow M$ is also a ring homomorphism.

Therefore $\operatorname{Spec}(\mathrm{gf}): \operatorname{Spec}(\mathrm{M}) \rightarrow \operatorname{Spec}(\mathrm{R})$ by 


$$
\begin{aligned}
& \operatorname{Spec}(\mathrm{gf})(\mathrm{Q})=\left(\mathrm{gf}^{-1}(\mathrm{Q}), \forall \mathrm{Q} \in \operatorname{Spec}(\mathrm{M})\right. \\
& =\left(\mathrm{f}^{-1} \mathrm{~g}^{-1}\right)(\mathrm{Q}) \\
& =\operatorname{Spec}(\mathrm{f})((\operatorname{Spec}(\mathrm{g})(\mathrm{Q})) \\
& \Rightarrow \operatorname{Spec}(\mathrm{gf})(\mathrm{Q})=(\operatorname{Spec}(\mathrm{f}) \operatorname{Spec}(\mathrm{g}))(\mathrm{Q}), \forall \mathrm{Q} \in \operatorname{Spec}(\mathrm{M}) \text {. } \\
& \Rightarrow \operatorname{Spec}(\mathrm{gf})=\operatorname{Spec}(\mathrm{f}) \operatorname{Spec}(\mathrm{g}) \\
& \text { Also, } \operatorname{Spec}\left(I_{R}\right)=I_{\text {Spec }(R)} \text {, where } I_{R}: R \rightarrow R \text { in } \mathbf{R} \text {. }
\end{aligned}
$$

Using Lemma 2.4, we say that the set of all subvarieties of A has a bijective correspondence with the set of all prime ideals of the ring $\mathbf{R}[\mathrm{A}]$, where $\mathbf{R}[\mathrm{A}]$ denotes the set of all polynomial functions on $\mathrm{A}$.

Let $\mathrm{h}^{\mathbf{R}[\mathrm{A}]}(\mathbf{R})=\operatorname{Hom}(\mathbf{R}, \mathbf{R}[\mathrm{A}])$.

We define for each $\mathrm{f}: \mathrm{R} \rightarrow \mathrm{T}$ in $\mathbf{R}, \mathrm{h}^{\mathbf{R}[\mathrm{A}]}(\mathrm{f})=\operatorname{Hom}(\mathrm{T}, \mathbf{R}[\mathrm{A}]) \rightarrow \operatorname{Hom}(\mathrm{R}, \mathrm{R}[\mathrm{A}])$ by

Theorem 3.4 $\mathrm{h}^{\mathrm{R}[\mathrm{A}]}: \mathbf{R} \rightarrow \mathbf{T}$ is a contravariant functor.

$$
\mathrm{h}^{\mathbf{R}[\mathrm{A}]}(\mathrm{f})(\alpha)=\alpha \text { o f, } \forall \alpha \in \operatorname{Hom}(\mathrm{T}, \mathbf{R}[\mathrm{A}]) \text {. }
$$

Proof: From [5], it follows

Thus we have two contravariant functors $\mathbf{S p e c}$ and $\mathbf{h}^{\mathbf{R}[\mathbf{A}]}$ from the category $\mathbf{R}$ to the category $\mathbf{T}$.

Now we have the following Theorems:

Theorem 3.5 For each algebraic set $\mathrm{A}$ in $\mathrm{K}^{\mathrm{n}}$, there is an equivalence $\Omega:\left(\mathbf{S p e c}, \mathbf{h}^{\mathbf{R}[\mathbf{A}]}\right) \rightarrow$ $\operatorname{Spec}(\mathrm{R}[\mathrm{A}])$, where (Spec, $\mathbf{h}^{\mathbf{R}[\mathbf{A}]}$ ) is the set of all natural transformations from the contravariant functor 'Spec' to the contravariant functor $\mathbf{h}^{\mathrm{R}[\mathrm{A}]}$.

Proof: Using Yoneda's Lemma, it follows.

Corollary 3.6 For each set $\mathrm{A}$ in Zarisky topology in $\mathrm{K}^{\mathrm{n}}$, there is an equivalence from the set of all natural transformations from the contravariant functor Spec to the contravariant functor $\mathbf{h}^{\mathbf{R}[\mathbf{A}]}$ to the Zarisky topology 'Spec(R[A])'

Proof: Using Lemma 2.4, Lemma 2.8, Theorem 3.1, Theorem 3.2, Theorem 3.3 and Theorem 3.4, it follows.

Theorem 3.7 For an affine $\mathrm{k}$-algebraic set $\mathrm{A}$ in $\mathrm{K}^{\mathrm{n}}$, the set of all $\mathrm{k}$-subvarieties in $\mathrm{K}^{\mathrm{n}}$ is equipotent to the set (Spec, $\mathbf{h}^{\mathbf{R}[\mathbf{A}]}$ ) of all natural transformations from the contravariant functor Spec to the contravariant functor $\mathbf{h}^{\mathbf{R}[\mathbf{A}]}$.

Proof: From [5], it follows.

Corollary 3.8 For each set A of the Zarisky topology in $\mathrm{K}^{\mathrm{n}}$ is equipotent to the set of all natural transformations from the contravariant functor $\mathbf{S p e c}$ to the contravariant functor $\mathbf{h}^{\mathbf{R}[\mathbf{A}]}$.

Proposition 3.9 The set (Spec, $\mathbf{h}^{\mathbf{R}[\mathbf{A}]}$ ) of all natural transformations from the contravariant functor Spec to the contravariant functor $\mathbf{h}^{\mathbf{R}[\mathbf{A}]}$ froms a Zarisky topology in $\mathrm{K}^{\mathrm{n}}$.

Proof: Using Theorem 3.1 and Theorem 3.7 and Corollary 3.8, it follows.

Proposition 3.10

i) The Zarisky topology in $\mathrm{K}^{\mathrm{n}}$ is not Hausdorff;

ii) The Zarisky topology in $\mathrm{K}^{\mathrm{n}}$ may not be $\mathrm{T} 1$ unless $\mathrm{K}=\mathrm{k}$

\section{Proof:}

i) Let $A$ be a k-variety in $K^{n}$. Then for any two nonempty open sets $U_{1}$ and $U_{2}$ in $A$ such that $U_{1} \cap U_{2} \neq \varnothing$, for if $U_{1} \cap U_{2}=\varnothing$, then $A=\left(A-U_{1}\right) \cup\left(A-U_{2}\right) \cup\left(U_{1} \cap U_{2}\right)$ i.e.,

$A=\left(A-U_{1}\right) \cup\left(A-U_{2}\right)$ and hence $A$ is the union of two affine k-algebraic sets $A-U_{1}$ and $A-U_{2}$ which are different from $\mathrm{A}$ i.e., $\mathrm{A}$ is not a k-variety in $\mathrm{K}^{\mathrm{n}}$. Therefore, if $\mathrm{x}$ and $\mathrm{y}$ are distinct points of $\mathrm{A}$ it is not possible to find disjoint neighbourhoods $U_{1}$ and $U_{2}$ of $x$ and $y$ respectively. This implies that $A$ is not a Hausdorff space. Therefore $\mathrm{K}^{\mathrm{n}}$ cannot be a Hausdorff space, because every subspace of a Hausdorff space is a Hausdorff space.

ii) If $\mathrm{k}=\mathrm{K}$, then any point of $\mathrm{K}^{\mathrm{n}}$ is an algebraic set and hence closed in the Zarisky topology. If $\mathrm{k} \neq \mathrm{K}$ and if $\left(\mathrm{x}_{1}, \mathrm{x}_{2}, \mathrm{x}_{3}, \ldots, \mathrm{x}_{\mathrm{n}}\right) \in \mathrm{K}^{\mathrm{n}}$ is not a zero of any polynomial in $\mathrm{k}\left[\mathrm{x}_{1}, \mathrm{x}_{2}, \mathrm{x}_{3}, \ldots, \mathrm{x}_{\mathrm{n}}\right]$, then the point $\left(\mathrm{x}_{1}, \mathrm{x}_{2}, \mathrm{x}_{3}, \ldots, \mathrm{x}_{\mathrm{n}}\right)$ is not a $\mathrm{k}$-algebraic set and hence not closed.

Corollary 3.11 Any open subset in the Zarisky topology in $\mathrm{K}^{\mathrm{n}}$ is a connected dense subset.

Proof: Using Proposition 3.10, it follows.

Corollary 3.12 Any open subset in the Zarisky topology in $\mathrm{K}^{\mathrm{n}}$ is connected. 


\section{References}

[1] Massey, W.S., (1984):- Algebraic topology; an introduction, Springer Verlag.

[2] Munkers, J.R.(1975):- Topology a first course. Prentice Hall Inc.

[3] Spanier, H. (1966):- Algebraic topology, Tata Mc-Graw-Hill Pub. Co. Ltd

[4] Adhikary, M.R., (1999):- Groups, Rings and Modules with Applications Universities Press, India.

[5] Rana, P.K. and Adhikari, A., (2000):- A representation of affine variety, Bull. Cal. Math. Soc., $8(1 \& 2) 21-24,2000$

[6] Rana, P.K.(2009):-A study of the group of covering transformation through functors, Bulletin Mathematique,33 (Lix) 2009(21-24);

[7] Rana, P.K.(2011):-A study of functors associated with rings on continuous functions, JIAM Vol.33(1), 2011, 73-78 